\title{
RESPOSTA DO FEIJOEIRO A DOSES DE FÓSFORO EM SOLO ARENOSO
}

\author{
RESPONSE OF COMMON BEAN TO PHOSPHORUS DOSES IN SANDY SOIL
}

\section{Enilson de Barros Silva ${ }^{1}$ José Carlos Fialho de Resende ${ }^{2}$ Welber Braga Rennó Cintra ${ }^{3}$}

\section{RESUMO}

A disponibilidade de fósforo é influenciada por vários atributos do solo que afeta a resposta das culturas à aplicação de P. Foi conduzido um experimento a campo, na Fazenda Experimental de Mocambinho (EPAMIG), na região do Projeto Jaíba, Norte de Minas Gerais em Areia Quartzosa (AQ) com os objetivos de avaliar a resposta do feijoeiro à aplicação de doses de $\mathrm{P}_{2} \mathrm{O}_{5}$ e estimar os níveis críticos de $\mathrm{P}$ no solo e nas folhas. Foram aplicadas quatro doses de $\mathrm{P}_{2} \mathrm{O}_{5}(0,35,70$ e $140 \mathrm{~kg}$ $\left.h \mathrm{a}^{-1}\right)$. Os tratamentos foram dispostos no delineamento de blocos casualizados, com cinco repetições. Usou-se a cultivar Carioca com uma população de 240.000 plantas por ha. Foram avaliadas o rendimento de grãos, o teor de $P$ no solo (Mehlich 1) e o teor foliar no ano 2000. O rendimento de grãos do feijão aumentou com as doses de $\mathrm{P}_{2} \mathrm{O}_{5}$, atingindo um rendimento de máxima eficiência econômica (MEE) de $3.821 \mathrm{~kg} \mathrm{ha}^{-1} \mathrm{com}$ a aplicação da dose de $98 \mathrm{~kg} \mathrm{ha}^{-1}$ de $\mathrm{P}_{2} \mathrm{O}_{5}$. O nível crítico de $\mathrm{P}$ no solo, associado à dose de MEE foi $15,7 \mathrm{mg} \mathrm{dm}^{-3}$ e para $P$ foliar foi $3,9 \mathrm{~g} \mathrm{~kg}^{-1}$.

Palavras-chave: Phaseolus vulgaris, nível crítico, solo, foliar, Areia Quartzosa.

\section{SUMMARY}

Phosphorus availabity is influenced by several attributes that affect the response of the crops to the $P$ application. The field experiment was conducted, on Mocambinho Experimental Farm (EPAMIG), "Projeto Jaíba” region, northern Minas Gerais, Brazil, on a Quartzipsament with the objetives of evaluating the response of common bean to $\mathrm{P}_{2} \mathrm{O}_{5}$ doses and critical levels for $P$ in soil and in leaves. Four $\mathrm{P}_{2} \mathrm{O}_{5}$ doses were applied $\left(0,35,70\right.$ and $\left.140 \mathrm{~kg} \mathrm{ha}^{-1}\right)$. The treatments were an followed by a randomized block design, with five replications. The cultivar "Carioca" with 240,000 populations of plants per ha was evaluated. The following observations were grains yield, soil $P$ content (Mehlich 1) and leaf content in year 2,000. The grains yield of common bean increased with $\mathrm{P}_{2} \mathrm{O}_{5}$ doses, attaining yield to optimum profit yield $(O P Y)$ of $3,821 \mathrm{~kg} \mathrm{ha}^{-1}$ with application of
$98 \mathrm{~kg} \mathrm{ha}^{-1}$ of $\mathrm{P}_{2} \mathrm{O}_{5}$. Phosphorus critical level in soil associated to OPY was $15.7 \mathrm{mg} \mathrm{dm}^{-3}$ and to phosphorus leaves was $3.9 \mathrm{~g} \mathrm{~kg}^{-1}$.

Key words: Phaseolus vulgaris, level critical, soil, leaf content, Quartzipsament.

\section{INTRODUÇÃO}

No Brasil, a necessidade de produção de alimentos para uma população crescente torna-se um desafio que poderá ser vencido com a exploração de novas áreas e com o aumento da produtividade. Nas duas situações, é fundamental a utilização racional dos diversos insumos agrícolas como corretivos e fertilizantes.

A produção é, em grande parte, o reflexo da fertilidade do solo ou das doses de fertilizantes aplicadas em uma lavoura. Esta é função da fertilidade dos solos, da eficiência dos adubos e de suas reações no solo, das necessidades das culturas e de fatores de ordem econômica. A disponibilidade de nutrientes dos solos, através de análises de solos, devem correlacionar-se com as quantidades absorvidas pelas plantas e, consequentemente, com a produção possibilitada pelo mesmo (ANGHINONI \& VOLKWEISS, 1984). Através das curvas de calibração, que relacionam a produção com a disponibilidade de nutrientes no solo, possibilitada por adubações conhecidas, pode-se estimar as adubações para obter produções máximas físicas e/ou econômicas (RAIJ, 1991).

\footnotetext{
${ }^{1}$ Engenheiro Agrônomo, Doutor, Empresa de Pesquisa Agropecuária de Minas Gerais (EPAMIG/CTNM), CP 12, 39440-000, Janaúba, MG. E-mail: ebsilva@ @ortecnet.com.br. Autor de correspondência.

${ }^{2}$ Engenheiro Agrônomo, Mestre, Empresa de Pesquisa Agropecuária de Minas Gerais (EPAMIG/CTNM), CP 12, 39440-000, Janaúba, MG.

${ }^{3}$ Técnico Agrícola, Empresa de Pesquisa Agropecuária de Minas Gerais (EPAMIG/FEMO), CP 12, 39468-000, Dist. Mocambinho, Jaíba, MG.
} 
A baixa disponibilidade de fósforo $(\mathrm{P})$, comum na maioria dos solos das regiões tropicais, transforma esse elemento em objeto de freqüentes estudos, que visam analisar os mecanismos reguladores do seu suprimento aos vegetais. O P movimenta-se predominantemente por difusão até à superfície das raízes, na qual ocorre sua absorção (MAHTAB et al., 1971). Os fatores genéticos da planta, a extensão do sistema radicular, o conteúdo de água e a concentração de $\mathrm{P}$ na solução do solo estão relacionados à difusão e, conseqüientemente, à absorção desse elemento pela planta (GOEDERT $\boldsymbol{e t}$ al., 1986).

Os fatores quantidade e poder-tampão de $\mathrm{P}$ que regulam o equilíbrio entre o P-lábil e o Psolução influenciam a concentração e quantidade de $\mathrm{P}$ na parte aérea das plantas (KAMPRATH \& WATSON, 1980, BAHIA FILHO, 1982, MUNIZ et al., 1985 e FONSECA, 1987). Os principais atributos do solo que refletem esses fatores são: teor de argila (LOPES \& COX, 1979 e NOVAIS \& KAMPRATH, 1979), matéria orgânica (BAHIA FILHO, 1982) e teor de água no solo (SILVEIRA \& MOREIRA, 1990).

$O$ feijoeiro tem baixa exigência em $P$, mas tem apresentado as maiores e mais freqüentes respostas quando aplicado à cultura (ROSOLEM, 1984). Alguns estudos demonstraram ausência de resposta à adubação fosfatada com $15 \mathrm{mg} \mathrm{dm}^{-3}$ de $\mathrm{P}$ no solo pelo Mehlich 1 (BARRIOS et al., 1970) ao passo que NOVAIS \& BRAGA FILHO (1971) encontraram decréscimo na produção quando aumentaram a dose de $\mathrm{P}$ aplicada em um solo com $24 \mathrm{mg} \mathrm{dm}^{-3}$ de P. Mas, outros verificaram respostas positivas como MASCARENHAS et al. (1967) com aplicação de $150 \mathrm{~kg} \mathrm{ha}^{-1}$ de $\mathrm{P}_{2} \mathrm{O}_{5}$, dose esta também encontrada por KORNELIUS et al. (1976) aplicando em um solo com $4 \mathrm{mg} \mathrm{dm}^{-3}$ de P.

CHAGAS et al. (1999) recomendam que para o feijoeiro, no qual é feito calagem, adubação, sementes fiscalizadas, 240.000 plantas por

ha, controle fitossanitário, tratamento de sementes e irrigação, a aplicação de P seja baseada no teor do solo em doses equivalentes a 90,70 e $50 \mathrm{~kg} \mathrm{ha}^{-1}$ de $\mathrm{P}_{2} \mathrm{O}_{5}$, quando a disponibilidade de $\mathrm{P}$ for baixo, médio e alto, respectivamente.

Os objetivos deste trabalho foram determinar o rendimento de grãos, dose de $\mathrm{P}$ e níveis críticos de $\mathrm{P}$ no solo e nas folhas do feijoeiro para máxima eficiência econômica.

\section{MATERIAL E MÉTODOS}

Um experimento de campo foi conduzido no Projeto Jaíba, município de Jaíba, Minas Gerais, Brasil, sendo a altitude da sede do município de $500 \mathrm{~m}$, a latitude de $15^{0} 23^{\prime} \mathrm{S}$, a longitude a $43^{0} 46^{\prime} \mathrm{W}$ e uma precipitação pluviométrica média anual de 800mm, sendo o clima classificado com Aw, segundo Köppen (ANTUNES, 1986).

Para a caracterização do solo foram coletadas amostras compostas na profundidade de 0 a $20 \mathrm{~cm}$ que, depois de secas ao ar e passadas em peneira de $2 \mathrm{~mm}$, foram analisadas química e fisicamente. Os resultados das análises para o solo classificado com Areia Quartzosa (AQ) são apresentadas na tabela 1 .

Os tratamentos foram quatro doses de $\mathrm{P}_{2} \mathrm{O}_{5}\left(0,35,70\right.$ e $\left.140 \mathrm{~kg} \mathrm{ha}^{-1}\right)$ aplicados no sulco de plantio na forma de superfosfato triplo. $O$ delineamento experimental utilizado foi de blocos casualizados com cinco repetições. Plantou-se feijão (Phaseolus vulgaris L.) cultivar Carioca, procurando-se obter 240.000 plantas por ha, população alcançada com o espaçamento de $0,50 \mathrm{~m}$ entre linhas e 12 plantas por metro linear. $\mathrm{O}$ plantio em 2000 foi feito em 14 de abril.

As adubações com $\mathrm{N}$ e $\mathrm{K}$ foram de, respectivamente, $50 \mathrm{~kg} \mathrm{ha}^{-1}(10 \mathrm{~kg}$ no plantio e $40 \mathrm{~kg}$ em cobertura aos 30 dias após a emergência) e 70kg ha $^{-1}$ de $\mathrm{K}_{2} \mathrm{O}$, nas formas de sulfato de amônio e cloreto de potássio, respectivamente. Com antecedência de 90 dias antes do plantio foram aplicados $400 \mathrm{~kg} \mathrm{ha}^{-1}$ de calcário dolomítico antes da aração em área total com a finalidade de fornecer $\mathrm{Ca}$ e Mg (ALVAREZ V. \& RIBEIRO, 1999). Foi também aplicado $2 \mathrm{~kg} \mathrm{ha}^{-1}$ de $\mathrm{Zn}$ na forma de sulfato de zinco.

O método de aplicação de água foi pelo sistema de aspersão com um lâmina diária de $3 \mathrm{~mm}$ com aspersores com vazão de $12,5 \mathrm{~mm} \mathrm{~h}^{-1}$. As parcelas tinham $2,5 \mathrm{~m}$ de largura (cinco linhas de

Tabela 1 - Características químicas e físicas do solo antes da implantação do experimento na profundidade de 0 a $20 \mathrm{~cm}$.

\begin{tabular}{|c|c|c|c|c|c|c|c|c|c|c|}
\hline $\mathrm{pH}$ água & $\mathrm{P}$ & $\mathrm{K}$ & $\mathrm{Ca}$ & $\mathrm{Mg}$ & $\mathrm{Al}$ & V & $\mathrm{MO}$ & Areia & Silte & Argila \\
\hline & \multicolumn{2}{|c|}{$--m g d^{-3}-$} & \multicolumn{3}{|c|}{$-----\mathrm{cmo} \ell_{\mathrm{c}} \mathrm{dm}^{-3}----$} & $--\%--$ & \multicolumn{4}{|c|}{-----------dag kg ${ }^{-1}$----------- } \\
\hline 5,7 & 2 & 21 & 1,4 & 0,2 & 0,1 & 56 & 0,7 & 85 & 5 & 10 \\
\hline
\end{tabular}

$\mathrm{pH}_{\text {água }}$ - Relação solo-água 1:2,5; P e K - Extrator Mehlich 1; Ca, Mg e Al - Extrator $\mathrm{KCl} 1 \mathrm{~mol} \ell^{-1} ; \mathrm{V}$ - Saturação em bases e MO - Teor de matéria orgânica determinado pelo método da oxidação do carbono por dicromato de potássio em meio ácido multiplicado por 1,724 . 
feijão) por $15 \mathrm{~m}$ de comprimento e correspondiam às doses de $\mathrm{P}_{2} \mathrm{O}_{5}$.

A análise de $\mathrm{P}$ disponível no solo foi feita em amostras retiradas no florescimento do feijoeiro, na profundidade de 0 a $20 \mathrm{~cm}$ através de amostras compostas por parcela. A determinação foi por extração com Mehlich 1. A amostragem para a determinação do teor de $\mathrm{P}$ nas folhas foi feita, segundo recomendação de MALAVOLTA $\boldsymbol{e t}$ al. (1997), ou seja, colhendo-se a primeira folha amadurecida a partir da ponta do ramo, no florescimento, 30 folhas por parcela. No extrato, obtido por digestão nitricoperclórica, foi dosado o teor de $\mathrm{P}$ total, por colorimetria, segundo metodologia descrita por MALAVOLTA et al. (1997).

Os dados de rendimento de grãos com umidade corrigida para $12 \%$, teor de P no solo e nas folhas foram submetidas à análise de variância e estudos de regressão, cujas equações foram ajustadas às variáveis em função das doses de $\mathrm{P}_{2} \mathrm{O}_{5}$ aplicadas. Os critérios para escolha dos modelos de regressão foram maior coeficiente de determinação, significância dos coeficientes de regressão até $5 \%$ de probabilidade pelo teste de $\mathrm{t}$ e significado biológico do modelo.

A partir das equações obtidas para produção, estimaram-se as doses correspondentes para máxima eficiência física (MEF) (máxima produção de grãos) e de máxima eficiência econômica (MEE) (máximo retorno econômico) que foram obtidas, respectivamente, igualando-se a primeira derivada da equação, correspondente à produção de grãos, ao valor zero e à relação de preços de $\mathrm{P}_{2} \mathrm{O}_{5}$ e de grãos de feijão.

Os níveis críticos de $\mathrm{P}$ disponível no solo e foliar foram estimadas, substituindo-se as doses para MEE, nas equações que relacionam as doses de $\mathrm{P}_{2} \mathrm{O}_{5}$ aplicadas com seus teores de $\mathrm{P}$ disponível e foliar.

\section{RESULTADOS E DISCUSSÃO}

O rendimento de grãos de feijão respondeu positivamente à aplicação de $\mathrm{P}$. Os resultados e as equações de regressão do rendimento de grãos colhidos sob o efeito de doses de $\mathrm{P}_{2} \mathrm{O}_{5}$, encontram-se na tabela 2 e figura 1.

O rendimento para máxima eficiência física (MEF) estimadas com a equação ajustada entre o rendimento de grãos e as doses de $\mathrm{P}_{2} \mathrm{O}_{5}$ aplicadas (Figura 1) foram obtidas com a dose de $104 \mathrm{~kg} \mathrm{ha}^{-1}$ de $\mathrm{P}_{2} \mathrm{O}_{5}$ e o rendimento para máxima eficiência econômica (MEE), com a relação média de $3.821 \mathrm{~kg} \mathrm{ha}^{-1}$, foi obtida com a dose de $98 \mathrm{~kg} \mathrm{ha}^{-1}$
Tabela 2 - Rendimento de grãos, teor de P disponível e foliar em função de doses de $\mathrm{P}_{2} \mathrm{O}_{5}$ em Areia Quartzosa.

\begin{tabular}{cccc}
\hline $\begin{array}{c}\text { Doses de } \mathrm{P}_{2} \mathrm{O}_{5} \\
\mathrm{~kg} \mathrm{ha}^{-1}\end{array}$ & $\begin{array}{c}\text { Rendimento de grãos } \\
\mathrm{kg} \mathrm{ha}^{-1}\end{array}$ & $\begin{array}{c}\mathrm{P}^{\text {Disponível }} \\
\mathrm{mg} \mathrm{dm}\end{array}$ & $\begin{array}{c}\mathrm{P}^{-3} \\
\mathrm{~g} \mathrm{~kg}^{-1}\end{array}$ \\
\hline 0 & 980,4 & 1,5 & 2,3 \\
35 & $2.847,9$ & 6,4 & 3,2 \\
70 & $3.356,3$ & 10,0 & 3,8 \\
140 & $3.532,9$ & 22,8 & 4,4 \\
Teste $\mathrm{F}$ & $83,11^{* *}$ & $296,54 * *$ & $13,88^{* *}$ \\
Dms $(5 \%)$ & 491,6 & 2,1 & 0,9 \\
\hline
\end{tabular}

${ }^{1 /}$ Extrator Mehlich $1 \mathrm{e} * *$ Significativo em nível de $1 \%$.

de $\mathrm{P}_{2} \mathrm{O}_{5}$ (Tabela 3). No período compreendido entre junho/1999 a junho/2000, considerando-se o rendimento de grãos nas relações de preços mais favoráveis e menos favoráveis (Tabela 3), as doses de $\mathrm{P}_{2} \mathrm{O}_{5}$ associadas à $\mathrm{MEE}$ variaram de 95 a $102 \mathrm{~kg}$ ha $^{-1}$ de $\mathrm{P}_{2} \mathrm{O}_{5}$, com dose média de $98 \mathrm{~kg} \mathrm{ha}^{-1} \mathrm{com}$ rendimento de grãos médio de $3.821 \mathrm{~kg} \mathrm{ha}^{-1}$ de feijão.

Pela análise inicial do teor de $\mathrm{P}$ na Areia Quartzosa, verifica-se que é considerado baixo por ALVAREZ et al. (1999), conforme observa-se na tabela 1. Portanto, a dose de $\mathrm{P}_{2} \mathrm{O}_{5}$ média para MEE está próxima da recomendação feita por CHAGAS et al. (1999) e abaixo da dose encontrada por MASCARENHAS $\boldsymbol{e t}$ al. (1967) e KORNELIUS $\boldsymbol{e} \boldsymbol{t}$ al. (1976) de $150 \mathrm{~kg} \mathrm{ha}^{-1}$ de $\mathrm{P}_{2} \mathrm{O}_{5}$.

Para diagnóstico da deficiência de $\mathrm{P}$, é importante realizar a análise do solo antecipadamente,

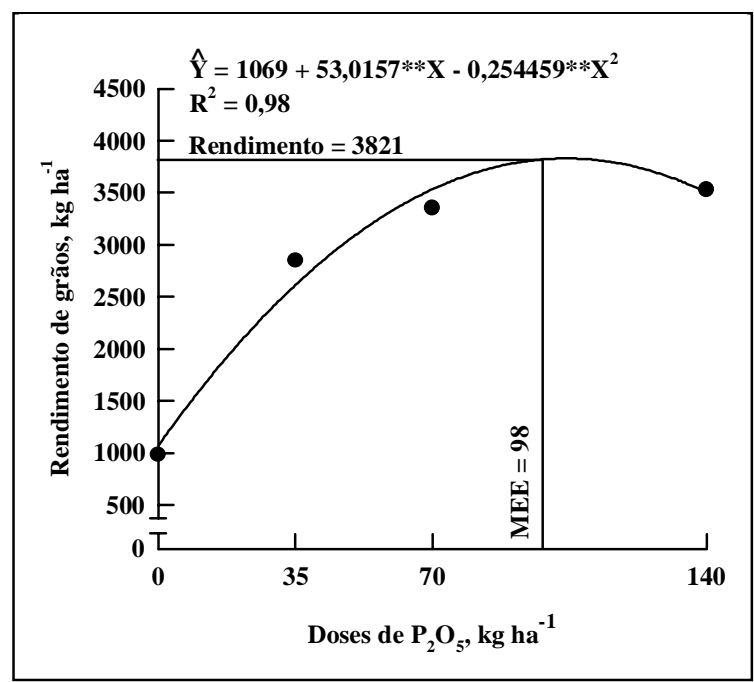

Figura 1 - Rendimento de grãos de feijão em função de doses de $\mathrm{P}_{2} \mathrm{O}_{5}$, em Areia Quartzosa e dose estimada para máxima eficiência econômica (MEE). (**significativo em nível de $1 \%$ pelo teste de $\mathrm{t}$ ) 
Tabela 3 - Doses de $\mathrm{P}_{2} \mathrm{O}_{5}$ aplicadas para máxima eficiência econômica (MEE), considerando o rendimento de grãos, em função da relação preço do $\mathrm{P}_{2} \mathrm{O}_{5} /$ preço de feijão.

Preço de feijão* Preço de $\mathrm{P}_{2} \mathrm{O}_{5} /$ Preço de feijão Dose de MEE

\begin{tabular}{ccc} 
por $60 \mathrm{~kg}$ & $\mathrm{R} \$ / \mathrm{kg}$ & $\mathrm{kg} \mathrm{ha}^{-1}$ \\
\hline $\mathrm{R} \$ 20,60$ & $0,4898^{1 /}$ & 95 \\
$\mathrm{R} \$ 33,01$ & $0,8726^{2 /}$ & 98 \\
$\mathrm{R} \$ 58,80$ & $1,3926^{3 /}$ & 102 \\
Média & & 98
\end{tabular}

* Preços obtidos junto a CONAB (2000), Brasília, DF

${ }^{1 /}$ Relação mínima observada entre junho/1999 a junho/2000

${ }^{2 /}$ Relação média observada entre junho/1999 a junho/2000

${ }^{3 /}$ Relação máxima observada entre junho/1999 a junho/2000

para fazer possíveis correções na adubação fosfatada do feijoeiro para minimizar prejuízos futuros no rendimento de grãos. Portanto, a partir da dose para máxima eficiência econômica (MEE), esta foi substituída na equação ajustada para o teor de $\mathrm{P}$ disponível em função das doses de $\mathrm{P}_{2} \mathrm{O}_{5}$ aplicadas no solo. Com este procedimento, registrou-se o nível crítico no solo de P. O modelo que melhor se ajustou ao teor de $\mathrm{P}$ disponível no solo foi o linear conforme se verifica na tabela 2 e figura $2 \mathrm{~A}$.

$\mathrm{O}$ nível crítico (NC) de $\mathrm{P}$ disponível foi de $15,7 \mathrm{mg} \mathrm{dm}^{-3}$ (Figura 2). Este teor crítico é considerado baixo dentro da classe de interpretação de disponibilidade de $\mathrm{P}$ de acordo com o teor de argila por ALVAREZ et al. (1999), mas BARRIOS et al. (1970), ao contrário deste trabalho não obteve resposta à adubação fosfatada com $15 \mathrm{mg} \mathrm{dm}^{-3}$ de $\mathrm{P}$ no solo. Por outro lado, NOVAIS \& BRAGA FILHO (1971) encontraram decréscimo no rendimento com teor superior ao obtido neste trabalho de $24 \mathrm{mg} \mathrm{dm}^{-3}$ de $\mathrm{P}$.

Em relação ao teor foliar de $\mathrm{P}$, utilizou-se o mesmo procedimento adotado para o $\mathrm{P}$ disponível, em que se estimou o nível crítico de $\mathrm{P}$ foliar (Figura 2). Observa-se que houve aumento linear do teor de $\mathrm{P}$ foliar com aumento das doses de $\mathrm{P}_{2} \mathrm{O}_{5}$ (Tabela $2 \mathrm{e}$ Figura 2).

$\mathrm{O}$ teor crítico de $\mathrm{P}$ foliar encontrado nas plantas de feijão, média das relações de preço, foi de $3,9 \mathrm{~g} \mathrm{~kg}^{-1}$ (Figura 2). Verifica-se, portanto, que o teor crítico está acima da faixa considerada adequada para o feijoeiro proposta por MALAVOLTA et al. (1997), de 2 a $3 \mathrm{~g} \mathrm{~kg}^{-1}$ de $\mathrm{P}$ e próximo do encontrado por FEITOSA et al. (1980) de 3,3g kg $\mathrm{gg}^{-1}$ em parcelas com alto rendimento de grãos $\left(3.100 \mathrm{~kg} \mathrm{ha}^{-1}\right) \mathrm{de}$ feijão irrigado.

As plantas cultivadas nos solos com os teores mais baixos de argila apresentam maiores teores de $\mathrm{P}$ na parte aérea. Esse maior teor foliar de $\mathrm{P}$ das plantas de feijão neste experimento (Tabela 1) e nível crítico foliar superior a MALAVOLTA $\boldsymbol{e t} \boldsymbol{a l}$. (1997) e FEITOSA $\boldsymbol{e t} \boldsymbol{a l}$. (1980) é o resultado do menor poder-tampão de fosfato desse solo, o que concorda com resultados obtidos por KAMPRATH \& WATSON (1980), BAHIA FILHO (1982), MUNIZ et al. (1985) e FONSECA (1987).

A irrigação associada ao menor podertampão de P da Areia Quartzosa pode ter favorecido a maior disponibilidade de $\mathrm{P}$ no solo e conseqüentemente maior absorção de $\mathrm{P}$ pelo feijoeiro resultando em elevado nível crítico de $\mathrm{P}$ foliar (Figura 2) e redução do nível crítico de $\mathrm{P}$ disponível no solo (Figura 2). Isso porque segundo KAMPRATH \& WATSON (1980), a maior concentração de $\mathrm{P}$ em solução, em solos arenosos, está normalmente associada a baixos valores de capacidade tampão. Logo, mesma quantidade de $\mathrm{P}$ absorvida pela planta haveria, proporcionalmente, maior redução da concentração de $\mathrm{P}$ em solução no solo arenoso do que no argiloso. Portanto, isto está relacionado ao baixo e alto nível crítico de $\mathrm{P}$ disponível no solo e foliar, respectivamente.

Figura 2 - Teor de $\mathrm{P}$ disponível (Mehlich 1) e foliar em função de doses de $\mathrm{P}_{2} \mathrm{O}_{5}$ e nível crítico (NC) relacionado com dose estimada para máxima eficiência econômica (MEE). (**significativo em nível de $1 \%$ pelo teste de $\mathrm{t}$ ) 


\section{CONCLUSÃO}

$\mathrm{O}$ rendimento de grãos de feijão aumentou com as doses de $\mathrm{P}_{2} \mathrm{O}_{5}$ aplicadas, atingindo valor máximo de $3.821 \mathrm{~kg} \mathrm{ha}^{-1}$. Essa dose de $\mathrm{P}_{2} \mathrm{O}_{5}$ de $98 \mathrm{~kg} \mathrm{ha}^{-1}$, dose esta para máxima eficiência econômica (MEE) e os níveis críticos de $\mathrm{P}$ disponível e foliar, associado à dose de MEE foram de $15,7 \mathrm{mg} \mathrm{dm}^{-3}$ e de $3,9 \mathrm{~g} \mathrm{~kg}^{-1}$, respectivamente.

\section{REFERÊNCIAS BIBLIOGRÁFICAS}

ALVAREZ V., V.H., NOVAIS, R.F., BARROS, N.F. de. $\boldsymbol{e} \boldsymbol{t} \boldsymbol{a l}$ Interpretação dos resultados das análises de solos. In: RIBEIRO, A.C., GUIMARÃES, P.T.G., ALVAREZ V., V.H. Recomendações para o uso de corretivos e fertilizantes em Minas Gerais. 5. Aproximação. Viçosa : CFSEMG, 1999. p. $25-32$.

ALVAREZ V., V.H., RIBEIRO, A.C. Calagem. In: RIBEIRO, A.C., GUIMAR ÃES, P.T.G., ALVAREZ V., V.H. Recomendações para o uso de corretivos e fertilizantes em Minas Gerais. 5. Aproximação. Viçosa : CFSEMG, 1999. p.43-60.

ANGHINONI, I., VOLKWEISS, S.J. Recomendações de uso de fertilizantes no Brasil. In: SIMPÓSIO SOBRE FERTILIZANTES NA AGRICULTURA BRASILEIRA, 1, 1984, Brasília. Anais Brasília : EMBRAPA/DEP, 1984. p.179-204.

ANTUNES, F.Z. Caracterização climática do Estado de Minas Gerais. Informe Agropecuário, v.12, n.138, p.9-13, 1986.

BAHIA FILHO, A.F.C. Índice de disponibilidade e fósforo em latossolos do Planalto Central com diferentes características texturais e mineralógicas. Viçosa-MG 1982. 178p. Tese (Doutorado em Solos e Nutrição de Plantas) - Curso de Pós-graduação em Solos e Nutrição de Plantas, Universidade Federal de Viçosa, 1982

BARRIOS, A., RODRIGUEZ, A.B., ORTEGA, S., $\boldsymbol{e}$ t al Resultados de ensayos de fertilización en caraota (Phaseolus vulgaris L.). Agronomia Tropical, Maracay, v.20, n.5, p.355-369, 1970 .

CHAGAS, J.M., BRAGA, J.M., VIEIRA, C. et al. Feijão. In: RIBEIRO, A.C., GUIMARÃES, P.T.G., ALVAREZ V., V.H. Recomendações para o uso de corretivos e fertilizantes em Minas Gerais. 5. Aproximação. Viçosa : CFSEMG, 1999. p.306-309.

CONAB. Companhia Nacional de Abastecimento. Indicadores da agropecuária Jun-Julho/2000. Brasília : Ministério da Agricultura e do Abastecimento, 2000. 50p.

FEITOSA, C.T., RONZELLI JÚNIOR, P., ALMEIDA, L.D.A. de et al. Adubação NP para o feijoeiro (Phaseolus vulgaris L.) na presença e na ausência de calcário. Revista Brasileira de Ciência do Solo, Campinas, v.4, p.156-159, 1980

FONSECA, D.M. Níveis críticos de fósforo em amostras de solos para o estabelecimento de Andropogon gayanus,
Brachiaria decumbens e Hyparrhenia rufa. Viçosa-MG, 1987. 146p. Dissertação (Mestrado em Solos e Nutrição de Plantas) - Curso de Pós-graduação em Solos e Nutrição de Plantas, Universidade Federal de Viçosa, 1987.

GOEDERT, W.J., SOUSA, D.M.G. de, LOBATO, E. Fósforo. In: GOEDERT, W.J. Solos dos cerrados: tecnologias e estratégicas de manejo. Brasília : EMBRAPA/CPAC, 1986. p.129-166.

KAMPRATH, E.J., WATSON, M.E. Conventional soil tests for assessing the phosphorus status of soils. In: KHASAWNEH, E.E., SAMPLE, E.C., KAMPRATH, E.J. (eds.) The role of phosphorus in agriculture. Madison : ASA/CSSA/SSSA, 1980. p.433-469.

KORNELIUS, E., SOBRAL, L.F., GOMES, J. de C., et al. Efeitos de doses de nitrogênio e fósforo na produção de feijão. In: CONGRESSO BRASILEIRO DE CIÊNCIA DO SOLO, 15, 1976, Campinas. Anais... Campinas : SBCS, 1976. p.203-205

LOPES, A.S., COX, F.R. Relação de características físicas, químicas e mineralógicas com fixação de fósforo em solos sob cerrados. Revista Brasileira de Ciência do Solo, Campinas, v.3, p.82-88, 1979.

MAHTAB, R.J., GODFREY, C.L., SWOBODA, A.B., et al Phosphorus diffusion in soils: the effect of applied P, clay content and water content. Proc Soil Sci Soc Am, Madison, v.35, p.393-397, 1971.

MALAVOLTA, E., VITTI, G.C., OLIVEIRA, S.A. Avaliação do estado nutricional das plantas. Piracicaba : POTAFOS, 1997. 201p.

MASCARENHAS, H.A.A., MIYASAKA, S., FREIRE, E.S., et al. Respostas do feijoeiro à adubação com N, P e K em solo orgânico de Ribeirão Preto. Bragantia, Campinas, v.26, n.57, 1967.

MUNIZ, A.S., NOVAIS, R.F., BARROS, N.F., et al. Nível crítico de fósforo na parte aérea da soja como variável do fator capacidade de fósforo do solo. Revista Brasileira de Ciência do Solo, Campinas, v.9, p.237-243, 1985.

NOVAIS, R.F., BRAGA FILHO, L.J. Aplicação de "tufito" e NPK na adubação do feijão, em um solo de Patos de Minas. Revista Ceres, Viçosa, v.18, n.98, p.308-314, 1971

NOVAIS, R.F., KAMPRATH, E.J. Fósforo recuperado em três extratores químicos como função do fósforo aplicado no solo e do "fator capacidade". Revista Brasileira de Ciência do Solo, Campinas, v.3, p.41-46, 1979.

RAIJ, B. van. Fertilidade do solo e adubação. Piracicaba : Ceres, 1991. 343p.

ROSOLEM, C.A. Nutrição e adubação feijoeiro. Piracicaba : POTAFOS, 1987. 91p.

SILVEIRA, P.M. da, MOREIRA, J.A.A. Resposta do feijoeiro a doses de fósforo e lâminas de água de irrigação. Revista Brasileira de Ciência do Solo, Campinas, v.14, p.63-67, 1990

Ciência Rural, v. 31, n. 6, 2001. 